\title{
Most Suitable Blade Inclination Angle for Multiphase Flow in Soybean Milk Machine
}

\author{
W. $\mathrm{Xu}^{1}$, L. $\mathrm{Li}^{1}$, H. Yin ${ }^{2}$ and H. S. Dou ${ }^{1 \dagger}$ \\ ${ }^{1}$ Faculty of Mechanical Engineering and Automation, Zhejiang Sci-Tech University, Hangzhou, Zhejiang \\ 310018, China \\ ${ }^{2}$ School of Mechanical and Electrical Engineering, Wenzhou University, Wenzhou, Zhejiang, 325035, China
}

†Corresponding Author Email: huashudou@yahoo.com

(Received May 06, 2021; accepted September 15, 2021)

\begin{abstract}
The blade inclination angle of soybean milk machine is a key geometric parameter for efficient crushing. For the purpose of obtaining optimal design, the gas-liquid two-phase flow field inside a soybean milk machine is simulated. The gas holdup from simulation is in agreement with the experiment. The simulation result shows that the lower blade A has a great influence on the internal flow field of soybean milk machine, while the upper blade B has a small influence on the flow field. As the angle $\left|\alpha_{A}\right|$ increases, the peak value of radial velocity decreases and moves to the interior of the cavity, so does the total pressure. When $\alpha_{A}$ changes from $-24^{\circ}$ to $-26^{\circ}$, the velocity vector at the bottom of the cavity changes from the connected state to the separated state, and the pressure difference between the up and the bottom surface of blade A becomes large. When $\alpha_{A}=-24^{\circ}$, the flow field has the strongest turbulent kinetic energy and dissipation. When $\alpha_{B}=28^{\circ}$, the pressure difference reaches the maximum. In summary, the best inclination angles are $\alpha_{\text {Aopt }}=-24^{\circ} \sim-26^{\circ}$ and $\alpha_{B o p t}=28^{\circ}$, respectively.
\end{abstract}

Keywords: Soybean milk machine; Two-phase flow; Blade inclination angle; Numerical simulation.

\section{INTRODUCTION}

Soybean milk has been loved by people for its unique taste and rich nutrition. With the improvement of living standards, people have higher and higher requirements on the taste and nutritional content of soybean milk. Soybean milk machine is a kind of small and fast equipment for making soybean milk.The crushing effect of soybean milk machine can directly affect the taste and nutritional content of soybean milk. Its various design parameters, including the shape of the cup, the blade installation height and the blade inclination angle, have a certain impact on the soymilk crushing efficiency.How to improve the design parameters of the soybean milk machine to improve the crushing effect of the soybean milk machine, thereby enhancing the taste and nutritional content of the soybean milk, has become a hot topic in the industry.

Wang et al. (2011) and Song et al. (2017) have carried out experimental or simulation research on the influence of blade inclination angle and the number ofguide ribs on the internal flow field of soybean milk machine. It is found that when the blade inclination angle is large and the angle between guide ribs is acute, the crushing effect is better.However, these studies were either experimental studies or simulation studies of single-phase media. There was no simulation study of two-phase media, and there were relatively few studies on the influence of the blade angle on the internal flow field of the soybean milk machine.Also as a rotary mixing machine, the principle of the agitator is similar to that of a soybean milk machine, so the research on the agitator can provide a certain reference for the soybean milk machine.Some scholars (Jia et al. 2020; Gradov et al. 2017; Zhang et al. 2018; Lane et al. 2002; Khopkar et al. 2005; Buffo et al. 2016; Wang et al. 2012; Kerdouss et al. 2006) have done a lot of researches on the agitator. Through the comparison of simulation results and experimental results, they found that CFD (Computational Fluid Dynamics) can effectively predict the characteristics of the gas-liquid two-phase flow field inside rotating machinery. The impeller is an important part of the agitator, its structure, installation angle, etc. can directly affect the flow field inside the agitator, and many scholars (Ren 
and Nan 2015; Wu et al. 2017; Shao et al. 2010; Zhou et al. 2013) have studied it.The Euler-Euler multiphase flow model has been used by many scholars (Jia et al. 2020; Li et al. 2010; Wang et al. 2012; Zhang et al. 2010; Li et al. 2017) to predict the two-phase flow field inside the agitator, and it is found that the simulation results are in good agreement with the experimental results.In the study of gas-liquid two-phase flow, gas holdup is an important research content. Previous scholars (Wu et al. 2001; Tian et al. 2017; Li et al. 2017; Prakash et al. 2019) studied a lot of content related to gas holdup and gas-liquid two-phase distribution. Through experiments and simulation comparisons, it was proved that CFD can effectively predict the gas-liquid two-phase distribution.Some of these scholars (Wu et al. 2001; Tian et al. 2017) deduced the relevant gas holdup rate equation by combining experiments with theory.

By summarizing the previous researches, it is found that it is meaningful to study the influence of the blade inclinationangle on the two-phase flow field inside the soybean milk machine.Considering the high rotation speed of the soybean milk machine and the high turbulence intensity, in this study, the Euler-Euler multiphase flow model and the RNG (Re normalization group) $k-\varepsilon$ turbulence model are used to numerically simulate the two-phase flow field inside the soybean milk machine.By analyzing the variations of the flow field characteristics under different blade inclination angles, the best blade inclination angle combination is found.

\section{GEOMETRIC MODEL AND MESH GENERATION}

\subsection{Geometric model ofsoybean milk machine}

The internal structure of the soybean milk maker is shown in Fig. 1, which mainly includes a lid, a cavity and a cutter,and the cutter is composed of four blades. In this paper, the blade with the downward inclination angle is defined as blade A, whichhas a negative inclination angle $\alpha_{A}$; the blade with the downward inclination angle is defined as blade B, which has a positive inclination angle $\alpha_{B}$. The parameters of the model are shown in Table 1 , andall the parameters are the real parameters provided by Joyoung Company Limited, a famous soybean milk machine company in China.The blade angles of the original model are $\alpha_{A}=-24^{\circ}$ and $\alpha_{B}=24^{\circ}$. In this paper, the control variable is adopted. First, the inclination angle of the B blade is kept unchanged, to study the influence of the inclination angle of blade A on the flow field, and obtain $\alpha_{\text {Aopt }}$ (the best inclination angle of the blade

A).Then, keeping $\alpha_{A}$ unchanged, study the influence of the inclination of blade B on the flow field, and obtain $\alpha_{B o p t}$ (the best blade B inclination angle).

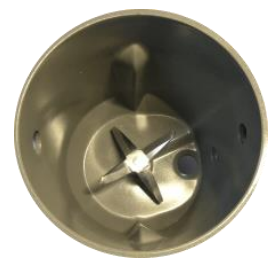

(a)

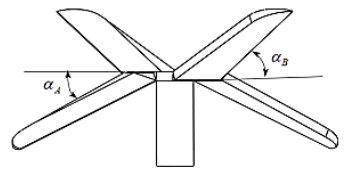

(b)

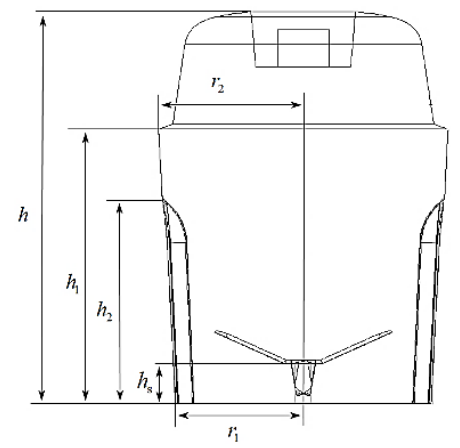

(c)

Fig. 1. Soybean milk machine model. (a) Cavity

photo; (b) Cutter model; (c) Overall model.

Table 1 Geometric dimensions of the model

\begin{tabular}{|l|l|}
\hline Parameter & Value \\
\hline Model total height, $h$ & $142.67 \mathrm{~mm}$ \\
\hline Cup body height, $h_{1}$ & $100 \mathrm{~mm}$ \\
\hline $\begin{array}{l}\text { The minimum radius of the cup } \\
\text { body, } r_{1}\end{array}$ & $48.02 \mathrm{~mm}$ \\
\hline $\begin{array}{l}\text { The maximum radius of the cup } \\
\text { body, } r_{2}\end{array}$ & $53.25 \mathrm{~mm}$ \\
\hline Spoiler height, $h_{2}$ & $75.27 \mathrm{~mm}$ \\
\hline Axis length, $h_{\mathrm{s}}$ & $16.3 \mathrm{~mm}$ \\
\hline Length of blade A, $l_{A}$ & $28 \mathrm{~mm}$ \\
\hline Length of blade B, $l_{B}$ & $28 \mathrm{~mm}$ \\
\hline Inclination angle of blade A, $\alpha_{A}$ & - \\
\hline Inclination angle of blade B, $\alpha_{B}$ & - \\
\hline $\begin{array}{l}\text { the best inclination angle of the } \\
\text { blade A, } \alpha_{A \text { Aopt }}\end{array}$ & - \\
\hline $\begin{array}{l}\text { the best inclination angle of the } \\
\text { blade B, } \alpha_{B o p t}\end{array}$ & - \\
\hline
\end{tabular}

The calculation region is divided into a rotating region and a stationary region. The rotating region and the stationary region are connected by interfaces, as shown in Fig.2 (a).Unstructured meshes are used to generate global meshes.Since the rotating region is an important part of the flow field and the accuracy of computation is important, the grids inside the impeller and on the blade surface are densified. Relatively speaking, the flow field in static domain is the secondary part. In order to save computing resources, the grid of thatdoes not need to be densified. Since the thickness of the 
front edge of blade is almost zero, the quality of the grid near it is poor, the minimum aspect ratio of the grid element is set to 0.3 , which can ensure the computational convergence.

\subsection{Mesh generation}

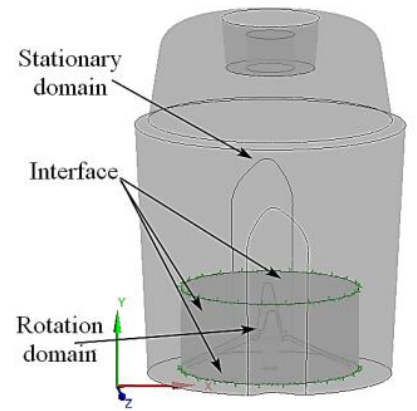

(a)

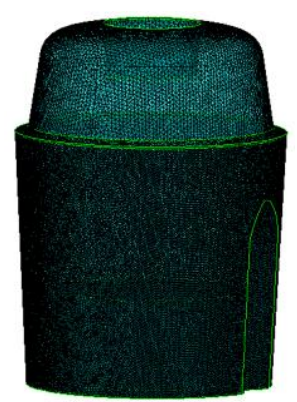

(b)

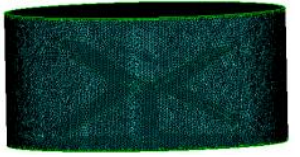

(c)
Fig. 2. Domain division and mesh generation. (a) Domain division; (b) Stationary domain grid; (c) Rotating domain grid.

\section{GOVERNING EQUATIONS AND NUMERICAL SIMULATION}

\subsection{Governing equations}

The three-dimensional N-S equations and Euler-Euler multiphase flow control equation are used to numerically do the simulation. The N-S equationsare:

$$
\begin{aligned}
& \rho\left(\frac{\partial u}{\partial t}+u \frac{\partial u}{\partial x}+v \frac{\partial u}{\partial y}+w \frac{\partial u}{\partial z}\right)= \\
& f_{x}-\frac{\partial p}{\partial x}+\mu\left(\frac{\partial^{2} u}{\partial x^{2}}+\frac{\partial^{2} u}{\partial y^{2}}+\frac{\partial^{2} u}{\partial z^{2}}\right) \\
& \rho\left(\frac{\partial v}{\partial t}+u \frac{\partial v}{\partial x}+v \frac{\partial v}{\partial y}+w \frac{\partial v}{\partial z}\right)= \\
& f_{y}-\frac{\partial p}{\partial y}+\mu\left(\frac{\partial^{2} v}{\partial x^{2}}+\frac{\partial^{2} v}{\partial y^{2}}+\frac{\partial^{2} v}{\partial z^{2}}\right) \\
& \rho\left(\frac{\partial w}{\partial t}+u \frac{\partial w}{\partial x}+v \frac{\partial w}{\partial y}+w \frac{\partial w}{\partial z}\right)= \\
& f_{z}-\frac{\partial p}{\partial z}+\mu\left(\frac{\partial^{2} w}{\partial x^{2}}+\frac{\partial^{2} w}{\partial y^{2}}+\frac{\partial^{2} w}{\partial z^{2}}\right)
\end{aligned}
$$

where, $\rho$ is the fluid density; $u, v, w$ are the velocity vectors in the $\mathrm{x}, \mathrm{y}$, and $\mathrm{z}$ directions respectively; $P$ is the pressure; $\mu$ is the dynamic viscosity coefficient; $f=\rho g$ is the external force per unit volume of the fluid.

The Euler-Euler multiphase flow equation is:

$$
\begin{aligned}
& \frac{\partial\left(\rho_{q} \alpha_{q} \phi_{q}\right)}{\partial_{t}}+\nabla \bullet\left(\rho_{q} \alpha_{q} V_{q} \phi_{q}\right)= \\
& \nabla \bullet\left(\Gamma_{\phi \bullet q} \nabla \phi_{q}\right)+\sum_{p=1}^{n}\left(\theta_{p q}+m_{p q} \phi_{p}-m_{q p} \phi_{q}\right)+S_{\phi \bullet q}
\end{aligned}
$$

where, $\rho_{q}$ represents the density of the $q$ phase; $\alpha_{q}$ represents the volume fraction of the $q$ phase; $\phi_{q}$ represents the dependent variable of the multiphase system; $V_{q}$ represents the $q$ phase velocity; $S_{\phi \bullet q}$ represents the original term; $n$ represents the number of phases; $m_{p q}$ represents the mass transfer from the $q$ phase to the $p$ phase; and $\theta_{p q}$ is the direct exchange of transported quantities (including momentum, energy and components).

\subsection{Boundary conditions}

Considering the high speed and strong turbulence intensity of soybean milk machine, the RNG $k-\varepsilon$ turbulence model and Euler-Euler multiphase flow model were used to calculate the internal flow field of soybean milk machine by CFD-CFX commercial software. The rotating speed of the blade is 2000 $\mathrm{rpm}$ and the rotating direction is counterclockwise from the top view. The rotating domain and the stationary domain are connected by interfaces. The frozen rotor model in multiple moving reference frames (MRF) is adopted (Thakur and Wright 2003; Sanaie-Moghadam et al. 2015; Guini, 2014).The main phase is water, and the gas phase is $25^{\circ} \mathrm{Cair}$; and the surface tension coefficient of gas-liquid is set to $0.073 \mathrm{~N} / \mathrm{m}$.According to the highest and lowest water level of this type of soymilk machine, taking the middle value as the initial condition, the initial volume fraction of water and air is obtained as 0.435 and 0.565 , respectively. The wall surface chooses a non-slip wall surface, without considering the influence of heat transfer and temperature, considering gravity and buoyancy.

\subsection{Grid independence verification}

Four sets of grids are used to verify the grid independence. The total pressure and static pressure with coordinates of $(30,30,30)$ are extracted and plotted. The results are shown in Fig. 3.It can be seen that when the number of grids is greater than or equal to 4.95 million, the total pressure and static pressure have little error.In addition, Fig. 4 shows the two-phase fluids distribution for the four sets of grids. Due to the instability of the gas-liquid two-phase flow, even under the same number of grids, the gas-liquid two-phase distribution cannot be completely consistent at different times. However, when the number of grids reaches 4.95 million, the bubble distribution above and below the 
blade is almost the same. In order to save computing resources, the set of 4.95 million grids is selected as the calculation grid in this paper.

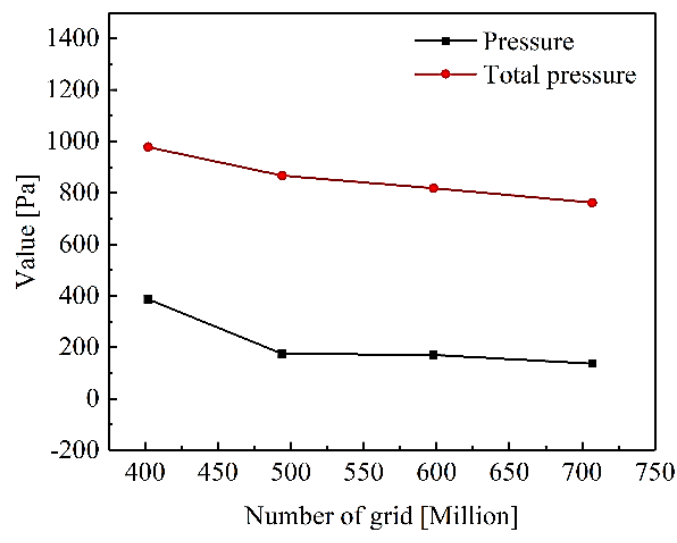

Fig. 3. Grid independence verification.

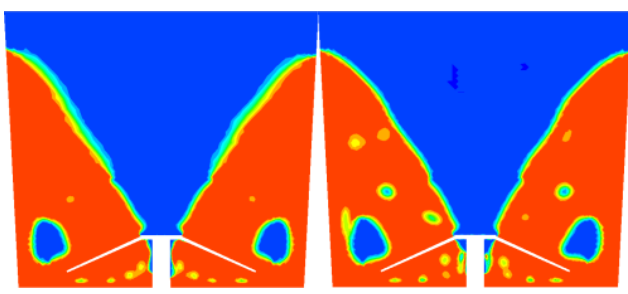

(a)

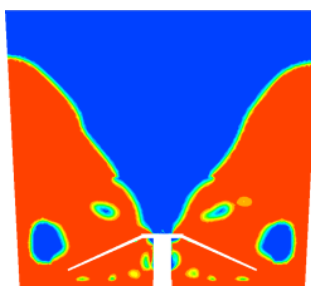

(c) (b)

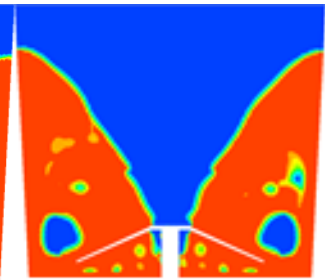

(d)
Fig. 4. Two-phase fluids distribution at the local $\mathrm{X}-\mathrm{Y}$ plane at different sets of grids.(a) 4 million; (b) 4.95 million; (c) 6 million; (d) 7.1 million.

The simulation results of this calculation method have been verified in previous studies, and it is found that the velocity of the experimental results and the one of simulation results are in good agreement ( $\mathrm{Li}$ et al. 2020). In this paper, the simulation effect of the calculation method on gas holdup is verified.The turbulence model, two-phase flow model and boundary conditions used in this paper are used to carry out numerical calculation for the agitator model in reference (Song et al. 2007). The comparison between the simulation results of gas content and the experimental results of that is shown in Fig.5. Due to the instability of gas-liquid two-phase flow and various errors of experiment and simulation, the values of experiment and simulation are not completely consistent, but the development trend is the same. It is proved that the calculation method used in this paper can effectively predict the two-phase flow field inside the soybean milk machine.

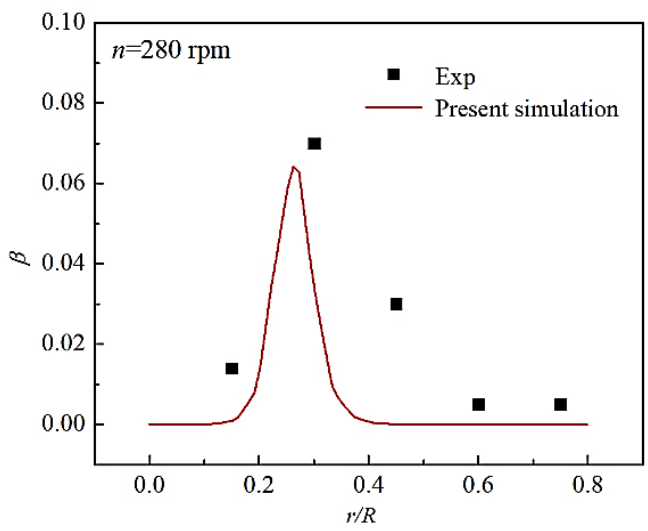

Fig. 5. Comparison of gas holdup between

simulation and experiment (Song et al.2007).

\section{RESULTS AND DISCUSSIONS}

\subsection{Influence of different $\alpha_{A}$ on the flow field}

\subsubsection{Two-phase fluids distribution of flow field}

Figure 6 shows the local gas-liquid two-phase fluids distribution contour on the $\mathrm{X}-\mathrm{Y}$ plane under different $\alpha_{A}$ at $\alpha_{B}=24^{\circ}$. It can be clearly found that $\alpha_{A}$ has a great impact on the gas-liquid two-phase fluids distribution. With the increase of $\left|\alpha_{A}\right|$, the sizes of bubbles at the bottom of the blade and above the tip of blade A first increase and then decrease. The angle $\alpha_{A}=-24^{\circ} \sim-26^{\circ}$ is a critical state. When $\left|\alpha_{A}\right|$ is less than $24^{\circ}$, there are a lot of bubbles at the bottom of the blade, and the bubbles above the tip gradually become large. When $\left|\alpha_{A}\right|$ is greater than $24^{\circ}$, the bubblesof the bottom of blade disappear, and the bubbles near the tip rise and become small.Thismay be related to the distance from the bottom to the blade A. When $\left|\alpha_{A}\right|$ is small, the distance from the bottom tothe blade A is relatively greater and closer to the upper gas phase of thecavity. With the high-speed rotation of the blade, the air in the upper part of the cavity is carried into the lower liquid phase; when $\left|\alpha_{A}\right|$ is larger, the blade $\mathrm{A}$ is far from the gas phase, so it is not easy to carry the air in the upper part of the cavity into the liquid phase Figure 7 is an analysis about gas holdup $\beta$ in the flow field. Figure 7(a) shows the variation process of blade surface gas content with $\left|\alpha_{A}\right|$. It can be found that with the increase of $\left|\alpha_{A}\right|$, the gas content on the blade surface first increases, and then a sudden change occurs at $\alpha_{A}=-24^{\circ} \sim-26^{\circ}$, from the maximum to 


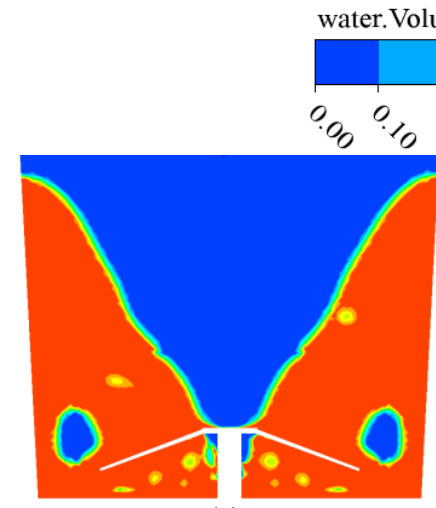

(a) $\alpha_{A}=-20^{\circ}$

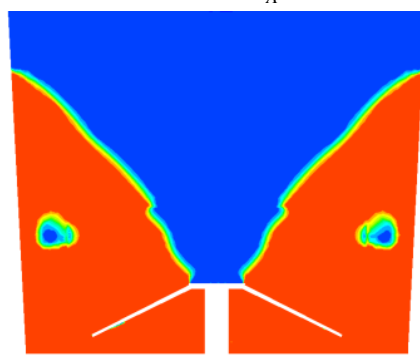

(d) $\alpha_{A}=-26^{\circ}$

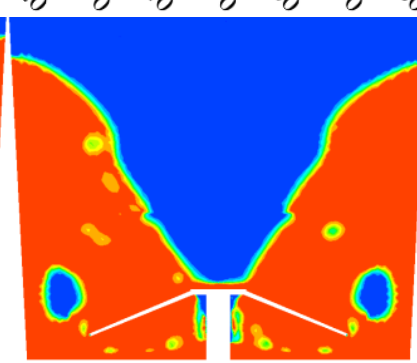

(b) $\alpha_{A}=-22^{\circ}$

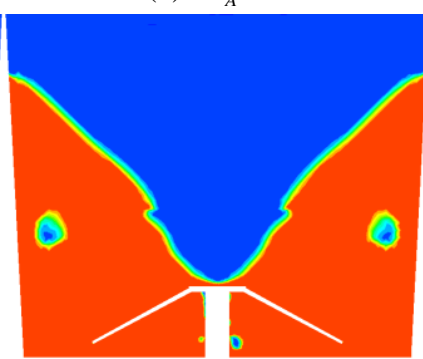

(e) $\alpha_{A}=-28^{\circ}$

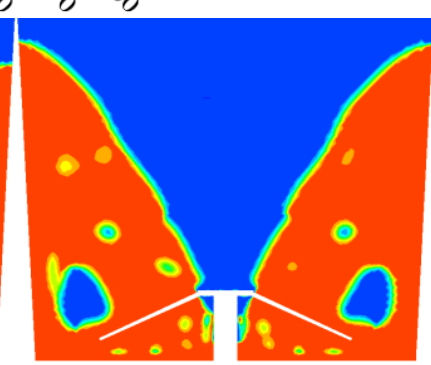

c) $\alpha_{A}=-24^{\circ}$

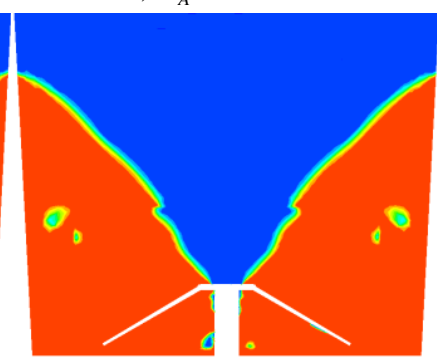

(f) $\alpha_{A}=-30^{\circ}$

Fig. 6. Two-phase fluids distribution at the local X-Y plane at different $\alpha_{A}$.

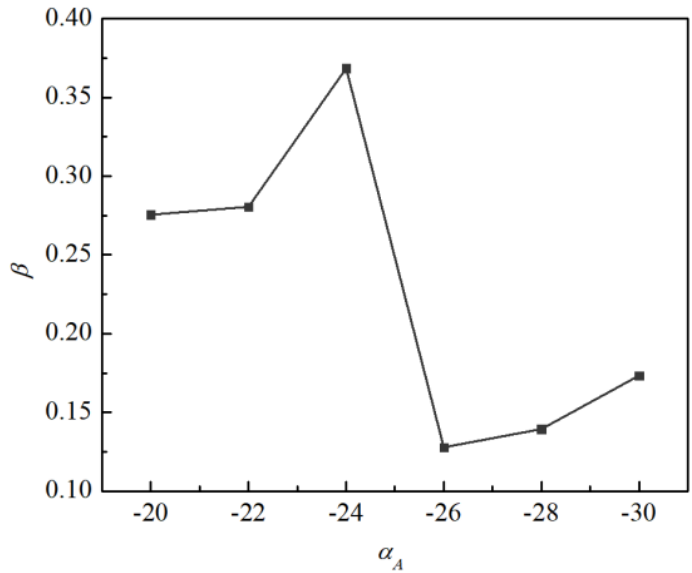

(a)

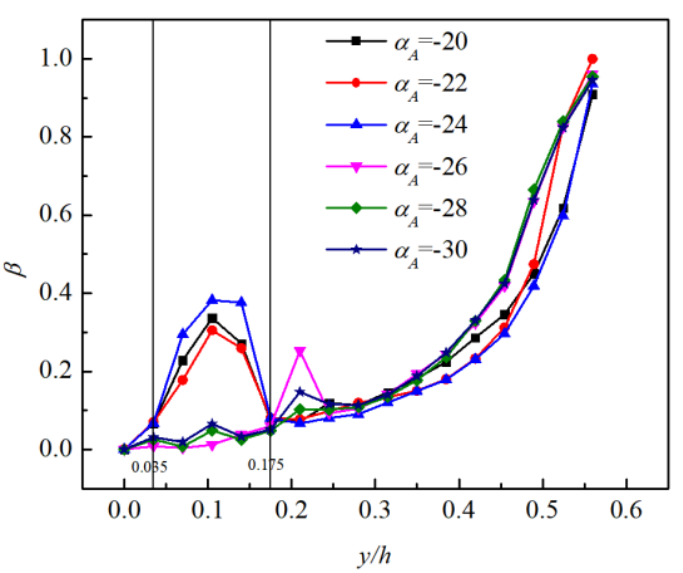

(b)

Fig. 7. Variation trend of gas content at different $\alpha_{A}$.(a) Variation of blade surface gas content; (b) Variation of the average gas content on different height planes.

the minimum, and then continues to increase. Figure 7(b) shows the variation process of the average gas content on different height planes under different $\alpha_{A}, y / h$ represents the ratio of the height of the plane to the total height of the model.

On the whole, $\beta$ gradually becomes larger as the height increases. But between $y / h=0.035 \sim 0.175$, at $\left|\alpha_{A}\right| \leq 24^{\circ}, \beta$ will increase first and then decrease, and the larger $\left|\alpha_{A}\right|$, the larger $\beta$; when $\left|\alpha_{A}\right| \geq 26^{\circ}, \beta$ does not vary much. This is also consistent with the two-phase distribution contour given earlier.

\subsubsection{Velocity vector distribution of the flow field}

Figure 8 shows the local velocity vector distribution on the X-Y plane under different $\alpha_{A}$ and $\alpha_{B}=24^{\circ}$.It can be found that when $\left|\alpha_{A}\right|$ is small, the flow field between the bottom of the blade and the corner of the cavity can connected, forming a counterclockwise rotating vortex. It can ensure that the fluid at the corners of cavity and the bottom of blade circulates to the blades, and the bean dregs will not remain in the corner of the cavity, thereby improving the crushing efficiency. With the increase 


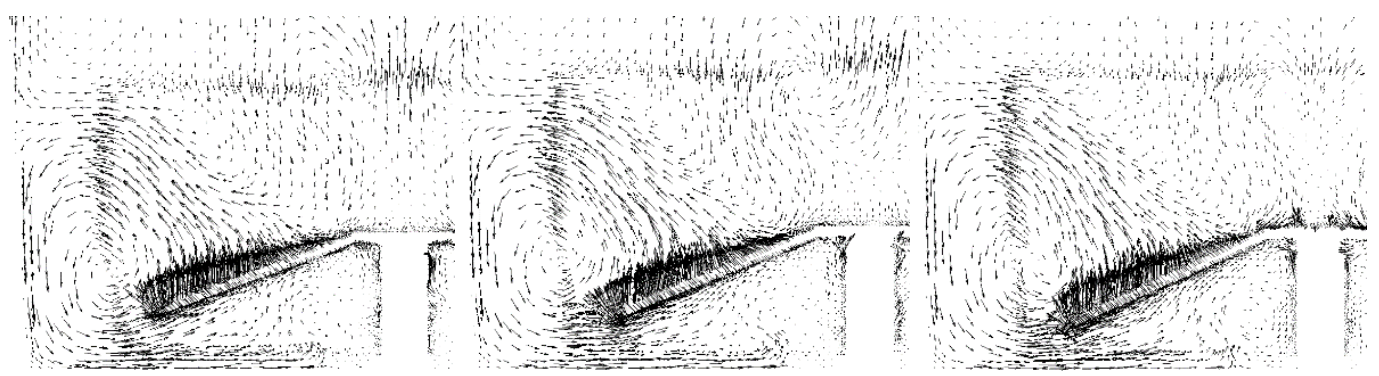

(a) $\alpha_{A}=-20^{\circ}$ (b) $\alpha_{A}=-22^{\circ}$ (c) $\alpha_{A}=-24^{\circ}$

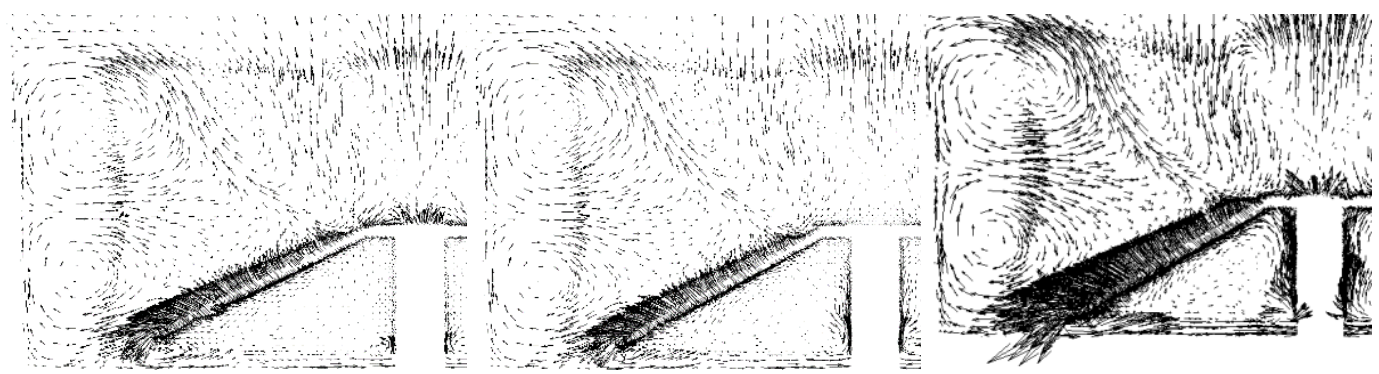

(d) $\alpha_{A}=-26^{\circ}$ (e) $\alpha_{A}=-28^{\circ}$ (f) $\alpha_{A}=-30^{\circ}$

Fig. 8. Local velocity vector on X-Y plane at different $\alpha_{A}$.

of $\left|\alpha_{A}\right|$, the flow field of the bottom of cavity gradually separates. The critical angle is $\alpha_{A}=-24^{\circ} \sim-26^{\circ}$. After that, the corners of cavity will form two self-circulating regions, forming a vortex rotating clockwise and counterclockwise from bottom to top. This will cause the bean dregs to self-circulate in the bottom corners, reducing the crushing efficiency.

Figure 9 shows the local velocity vector distribution on the Y-Z plane. It can be seen that when $\left|\alpha_{A}\right|$ is small, the flow field circulation at the corner of the cavity and the bottom of the blade is good. With $\left|\alpha_{A}\right|$ increasing, due to the Coanda effect, a row of very small vortices will appear near the bottom wall of the cavity. This causes the bean dregs to form a self-circulation area at this position, which reduces the probability of contact between the bean dregs and the blade. The critical value of the angle is $\alpha_{A}=-24^{\circ} \sim-26^{\circ}$.

A sampling line parallel to the $\mathrm{X}$ axis at the height of $y / h=0.14$ on the X-Y plane is taken to analyze the

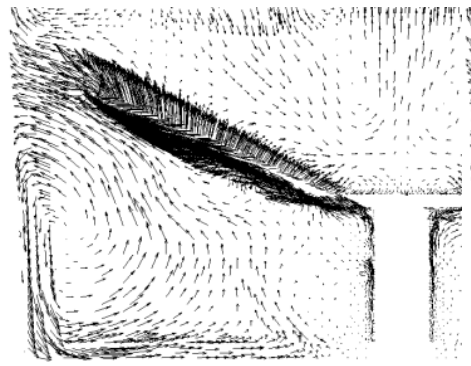

(a) $\alpha_{A}=-20^{\circ}$

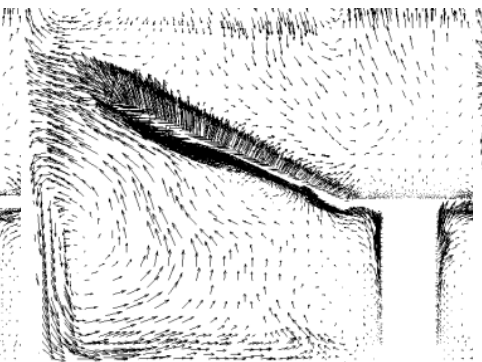

(b) $\alpha_{A}=-22^{\circ}$

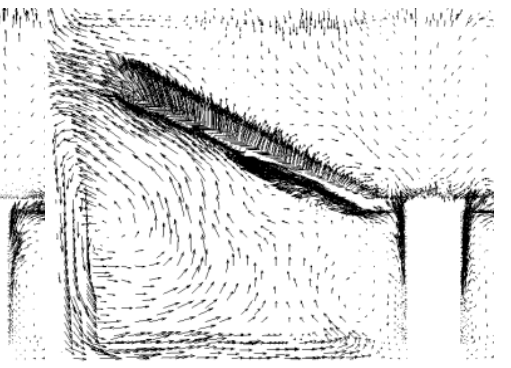

(c) $\alpha_{A}=-24^{\circ}$

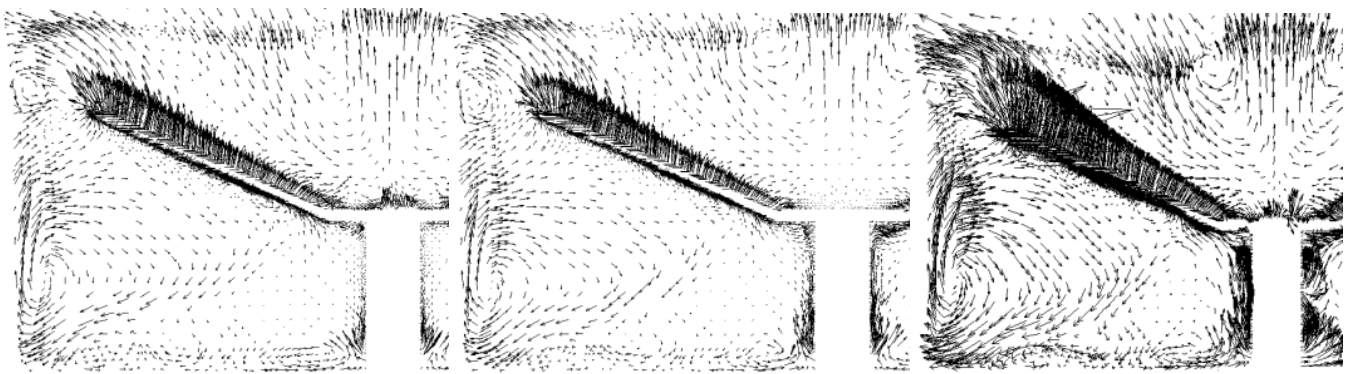

(d) $\alpha_{A}=-26^{\circ}$

(e) $\alpha_{A}=-28^{\circ}$

(f) $\alpha_{A}=-30^{\circ}$

Fig. 9. Local velocity vector on Y-Z plane at different $\alpha_{A}$. 
radial distribution of the flow field. Figure 10 shows the variation of various flow field characteristics on the line, and $x / r_{1}$ represents the ratio of the coordinate in the $\mathrm{X}$-axis direction on the line to the minimum radius of the cavity $r_{l}$. From Fig. 10 (a) and (b),it can be found that the velocity distribution has a strong symmetry, which is the smallest at the center and wall of the cavity. From the center to the wall of cavity, the velocity distribution first increases and then decreases. The peak velocity will move to the center of cavity with $\left|\alpha_{A}\right|$ increasing and the peak velocity will gradually decrease. The velocity of a mass point in the flow field is affected by the velocity of the fluid near it and the blade. As $\left|\alpha_{A}\right|$ increases, the height of A blade decreases, so the peak velocity at the same height will decrease and move to the center of cavity. The variation of the total pressure is basically the same as the velocity, but it should be noted that, unlike the speed, the total pressure maintains a minimum value at $x / r_{1} \approx \pm 0.1$ near the center of the cavity. With reference to Fig. 5, it can be found that this may be owing to in this region, the part at a height of $y / h=0.14$ is completely in the gas phase; regardless of the compressibility of the gas, the total pressure on the same height plane of the gas phase remains unchanged.

\subsubsection{Total pressure distribution across the blade}

Due to symmetry, Fig. 11 only shows the local total pressure contour near single blade. Column (a) represents the contour of total pressure near blade A, and column (b) represents the contour of total pressure near blade B. It can be seen from column (a) that with the increase of , the total pressure on the pressure surface (upper surface) of the blade A increases, while the one on the suction surface (lower surface) decreases, and the low pressure zone gradually moves from the tip of blade $\mathrm{A}$ to the shaft of blade. On the contrary, with the increase of $\left|\alpha_{A}\right|$, it can be seen from column (b) that the total pressure on the suction surface of the blade B gradually increases, and the low pressure area moves to the tip of the blade; the total pressure on the pressure surface does not change much. Total pressure is related to velocity and static pressure, so this phenomenon will be analyzed in combination with velocity in the following.

Because blade A is close to the bottom of the cavity, the pressure difference nearby that has a much greater impact on the crushing effect than blade B, and only the pressure difference across the blade A is analyzed quantitatively in this study. Figure 12 shows the quantitative analysis of the total pressure change across the blade $\mathrm{A}$ on the $\mathrm{X}-\mathrm{Y}$ plane. Figure 12(a) and (b) represent the total pressure changes on two sampling lines $l_{1}$ and $l_{2}$ parallel to Y-axis and $d_{1}$ and $d_{2}$ away from Y-axis, respectively. The interruption part is due to the flow field inside of the blade can't be collected, which is an objective fact. It can be found that the total pressure through the blade will vary abruptly, which is also due to sampling lines passing through the suction surface and pressure surface of the blade. Taking Fig. 12(a) as an example, it can be found that the total pressure of the suction surface first decreases and then increases with $\left|\alpha_{A}\right|$ increasing, and reaches the minimum value around $\alpha_{A}=-24$.The total pressure of the pressure surface first increases and then decreases with $\left|\alpha_{A}\right|$ increasing, and reaches the maximum value around $\alpha_{A}=-26$.Therefore, the maximum value of pressure difference should be at $\alpha_{A}=-24^{\circ} \sim-26^{\circ}$.

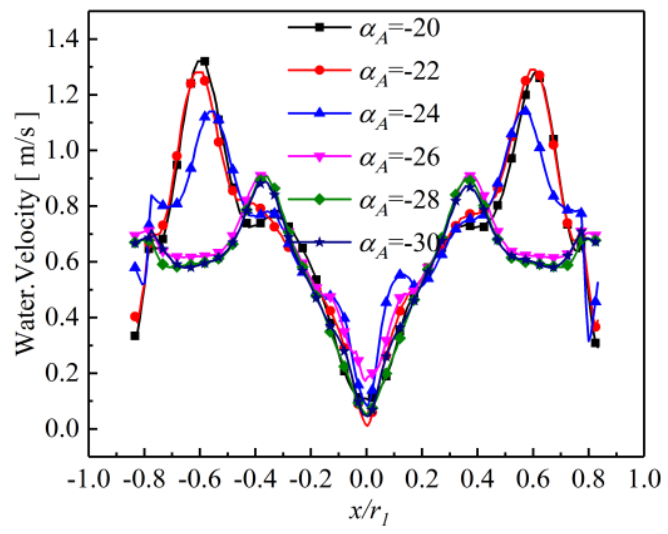

(a)

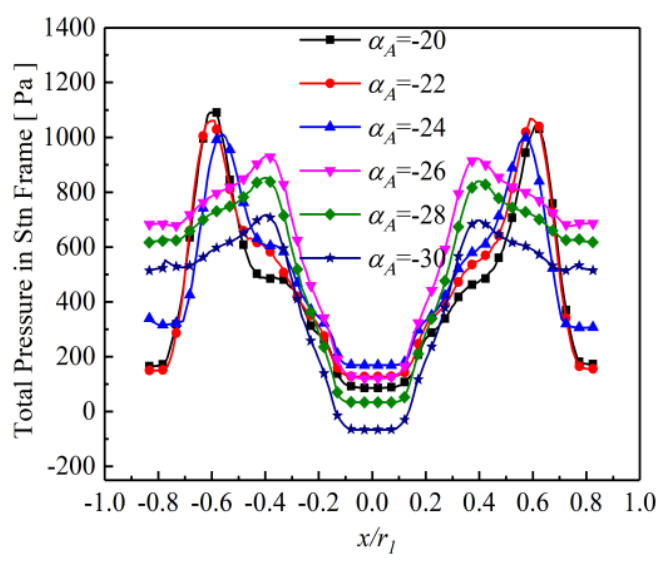

(b)

Fig. 10. Radial variation of velocity and total pressure at different $\alpha_{A}$. (a) Radial variation of velocity; (b) Radial variation of total pressure.

In Fig. 12(a) and (b), the total pressure of the suction surface has a small rebound with the increase of height, especially in (b). In order to analyze this phenomenon, the static pressure and velocity distributions on $l_{2}$ are shown in Fig. 12 (c) and (d), respectively. It can be seen that the static pressure on the suction surface is the lowest, but the velocity close to the suction surface has a rebound. This is because the velocity of a particle in the flow field is affected by the speed of the blade and the particle near it. The closer to the blade, the more the velocity of flow field is affected by the blade speed. The total pressure is composed of static pressure and dynamic pressure, so the total pressure of the suction surface has a certain increase. 
Total Pressure in
Stn Frame
\begin{tabular}{|l|}
\hline $4.000 \mathrm{e}+003$ \\
$-3.400 \mathrm{e}+003$ \\
$-2.800 \mathrm{e}+003$ \\
$-2.200 \mathrm{e}+003$ \\
$-1.600 \mathrm{e}+003$ \\
$-1.000 \mathrm{e}+003$ \\
$-4.000 \mathrm{e}+002$ \\
$-2.000 \mathrm{e}+002$ \\
$-8.000 \mathrm{e}+002$ \\
$-1.400 \mathrm{e}+003$ \\
$-2.000 \mathrm{e}+003$
\end{tabular} [Pa]

$$
\alpha_{A}=-20^{\circ}
$$$$
\alpha_{A}=-22^{\circ}
$$$$
\alpha_{A}=-22^{\circ}
$$

$$
\alpha_{A}=-24^{\circ}
$$

$\alpha$

$$
\alpha_{A}=-24^{\circ}
$$

$\alpha_{A}=-26^{\circ}$

$\alpha_{A}=-26^{\circ}$

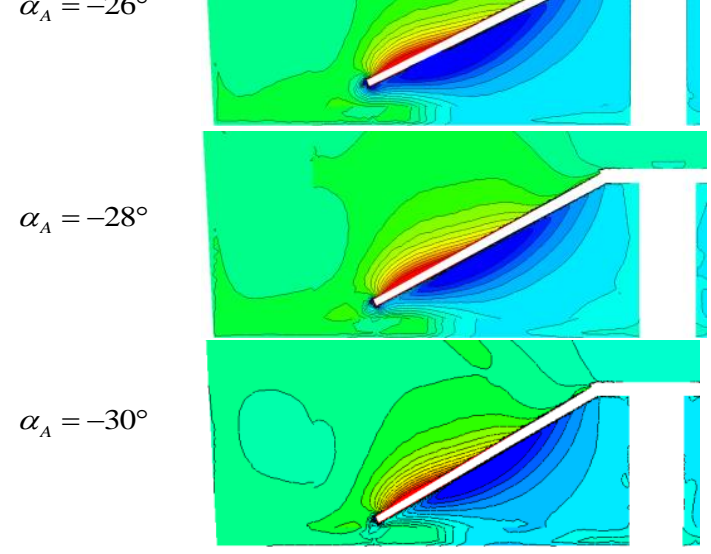

(a) Blade $\mathrm{A}$

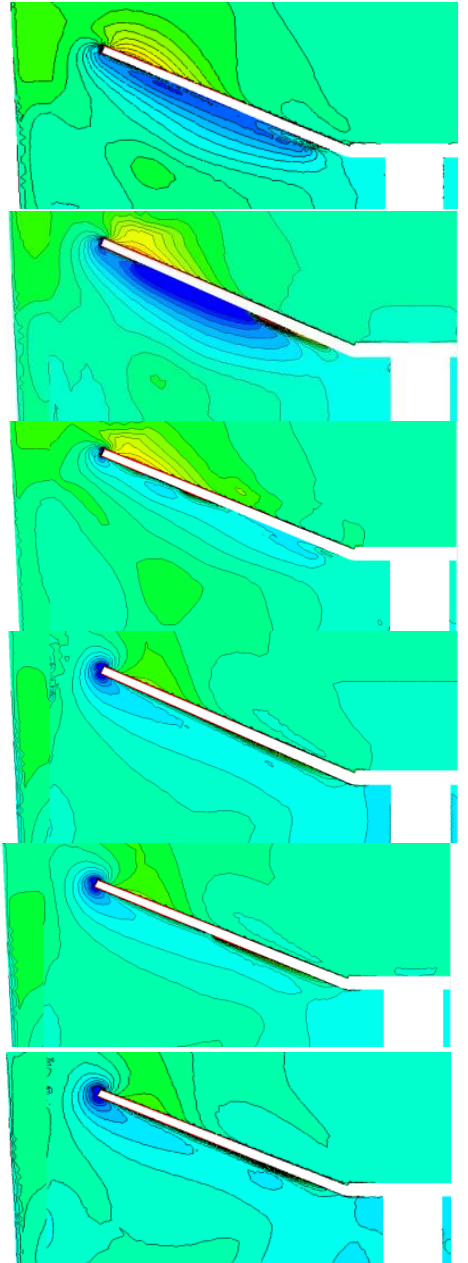

(b) Blade B

Fig. 11. Contour of total pressure distribution near blade at different $\alpha_{A}$.

Figure 12(e) shows the variation of the maximum pressure difference of $l_{1}$ and $l_{2}$ and its average value with $\alpha_{A}$.It can be clearly found that as $\left|\alpha_{A}\right|$ increases, the average pressure difference first increases and then decreases. The angle $\alpha_{A}=-24^{\circ} \sim-26^{\circ}$ is the critical angle range where the pressure difference varies suddenly. The greater the pressure difference, the greater the suction force of the blade on the particles at the bottom. Therefore, considering only the pressure difference, the inclination angle of the blade A should be taken $\alpha_{A}=-24^{\circ} \sim-26^{\circ}$, which can increase the contact probability of the soybean and blade, thereby improving the crushing efficiency.

\subsubsection{Variation of circumferential flow characteristics}

Take a circle with a radius of $37.5 \mathrm{~mm}$ on the height plane of the shaft as a sampling line to analyze the circumferential characteristics of the flow field. Figure 13 shows the variation of various fluid characteristics at the sampling line. It can be found that the existence of guide ribs has a great influence on the characteristics of the flow field. With the increase of $\left|\alpha_{A}\right|$, the velocity increases gradually, but the amplitude is not very large. When the fluid particle flows by guide rib, the velocity will vary from small to large and then to small. This is because under the condition of constant flow rate, due to the protrusion of the guide rib, the flow channel becomes small, the flow velocity increases, and the velocity after flowing by the guide ribs decreases again. The total pressure first increases and then decreases, and reaches the maximum around $\alpha_{A}=-26^{\circ}$.After the guide ribs, the total pressure varies from large to small and then to large. The trend of TKE(turbulent kinetic energy)variation is similar to that of the total pressure, except that it reaches the maximum around $\alpha_{A}=-24^{\circ}$, and TKE varies from the maximum to the minimum after passing by the guide ribs. The distribution of TED (turbulence eddy dissipation) is very chaotic, and it reaches the maximum around $\alpha_{A}=-24^{\circ}$ in general. 


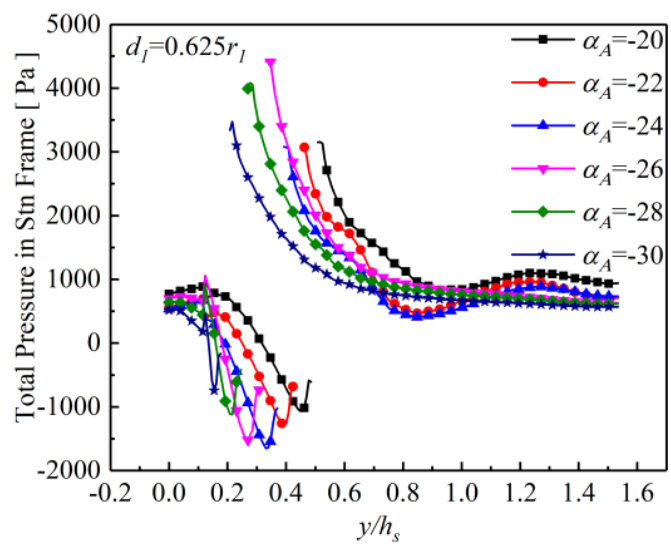

(a)

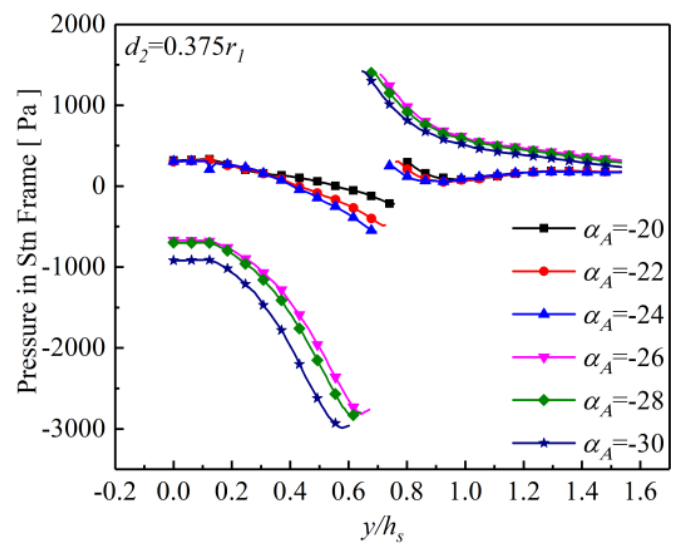

(c)

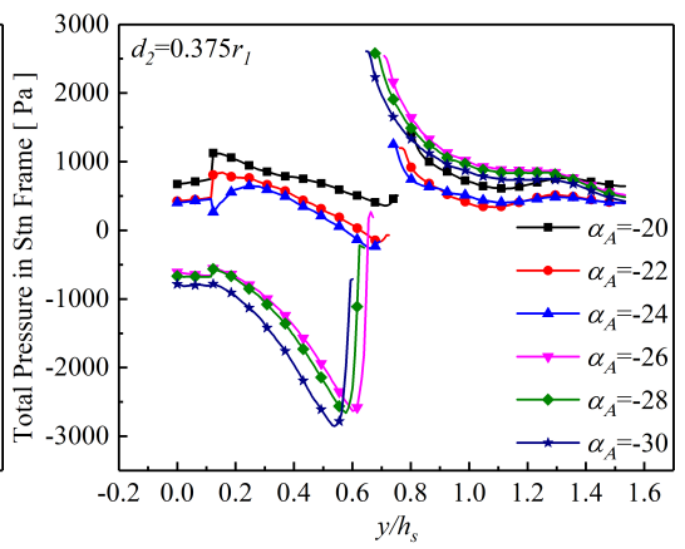

(b)

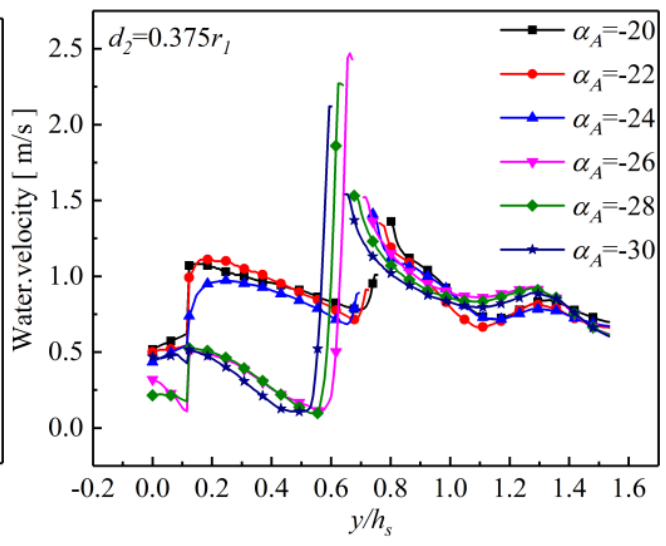

(d)

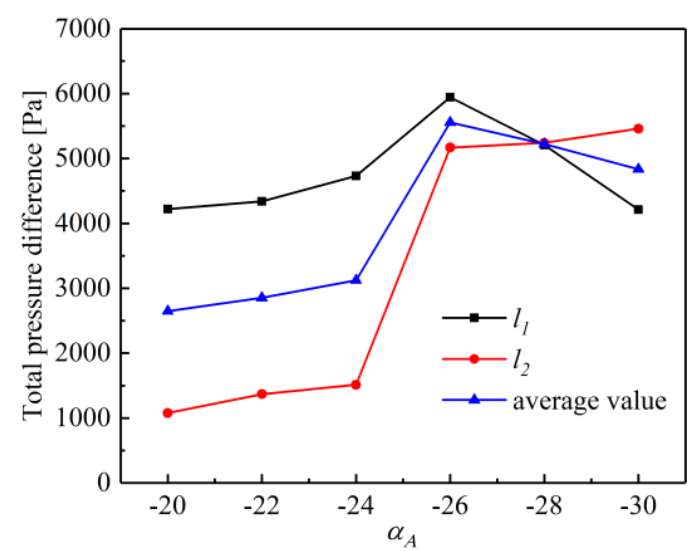

(e)

Fig. 12. Quantitative analysis of total pressure near $A$ blade at different $\alpha_{A}$. (a) Total pressure

variation on $l_{l}$; (b) Total pressure variation on $l_{2}$; (c) Static pressure variation on $l_{2}$; (d) Variation of water velocity on $l_{2}$; (e) Variation of the maximum pressure difference of $l_{1}$ and $l_{2}$ and its average value.

By analyzing the influence of $\alpha_{A}$ on the characteristics of the gas-liquid two-phase flow inside the soybean milk machine, it can be found that in the model studied in this paper, the best $\alpha_{A}$ should be $\alpha_{\text {Aopt }}=-24^{\circ} \sim-26^{\circ}$. 


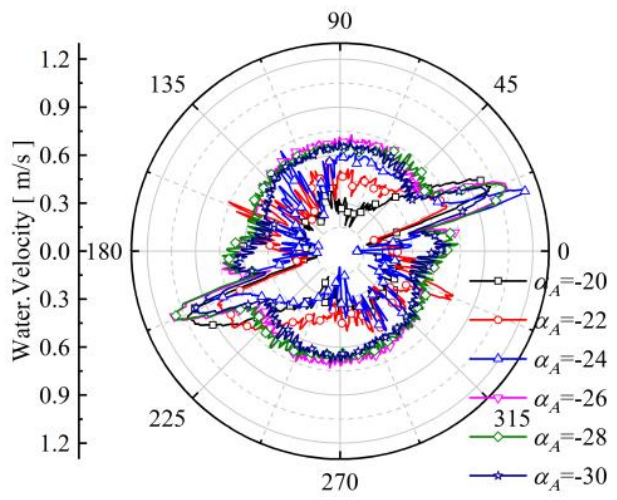

(a)

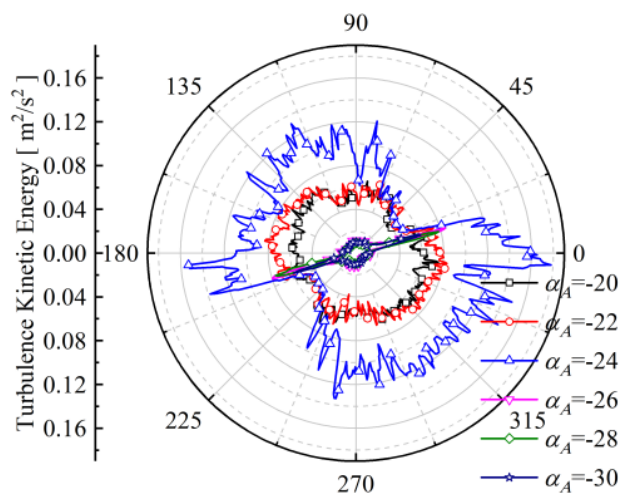

(b)

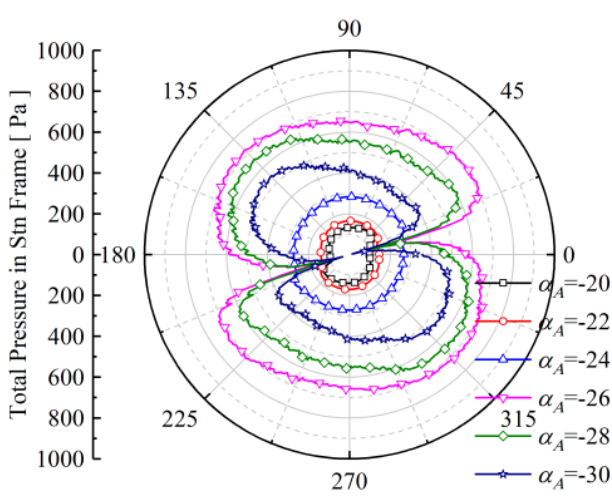

(b)

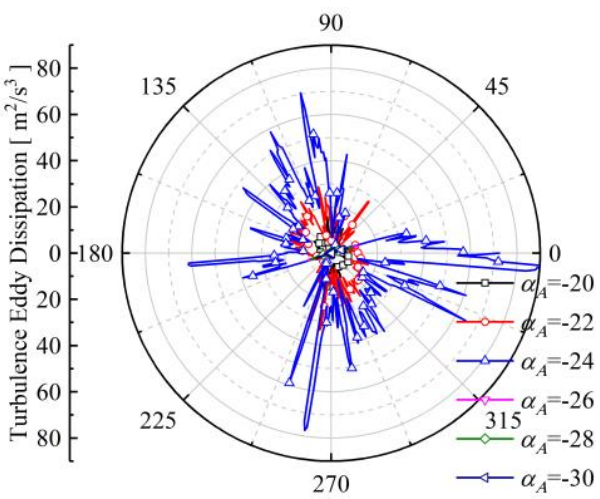

(d)

Fig. 13. Circumferential variation of various flow characteristics at different $\alpha_{A}$.(a) Circumferential variation of velocity; (b) Circumferential variation of velocity; (c) Circumferential variation of TKE; (d) Circumferential variation of TED.

\subsection{Influence of $\alpha_{B}$ on the flow field}

\subsubsection{Two-phase fluids distribution of flow field}

Figure 14 shows the influence of different $\alpha_{B}$ on the gas-liquid two-phasefluids distribution on the $\mathrm{X}-\mathrm{Y}$ plane at $\alpha_{A}=-24^{\circ}$. It can be found that only the change of $\alpha_{B}$ does not have much effect on the gas-liquid two-phase distribution of the flow field below the blade A, but it has a certain impact on the gas-liquid fluids distribution above the blade A.It can be seen that with the increase of $\alpha_{B}$, the gas holdup above the blade A has a certain increase. This is due to the increase of $\alpha_{B}$, the blade B is closer to the gas phase, and it is easier to carry the gas phase into the nearby liquid phase with the rotation of the blade.

\subsubsection{Velocity vector andtotal pressure distribution near blade}

It can be seen from Fig. 15 that only varying $\alpha_{B}$ has little effect on the velocity vector at the bottom of soybean milk machine.Therefore, the following is only a quantitative study of the influence of different $\alpha_{B}$ on the flow field.

Since the blade A is closer to the bottom of the cavity, the pressure difference nearby has a greater impact on the crushing effect of the soybean milk machine, Fig. 16 shows a quantitative analysis of the total pressure across the blade A at different $\alpha_{B}$.It can be found that as $\alpha_{B}$ increases, the average pressure difference near blade A first increases and then decreases, reaching the maximum near $\alpha_{B}=28^{\circ}$, which is most conducive to crushing soybeans.

According to the aspects discussed above, the best angle of inclination of blade B should be around $\alpha_{\text {Bopt }}=28^{\circ}$.

\section{CONCLUSIONS}

The gas-liquid two-phase flow field inside a soybean milk machine is simulated. The results show that the blade inclination angle of the soybean milk machine has a great impact on its internal two-phase flow field. The most suitable blade inclination angle formultiphase flow in the soybean 


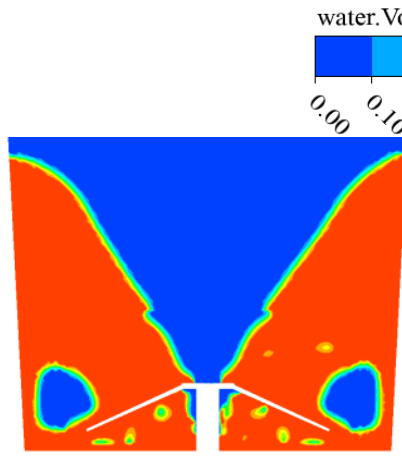

(a) $\alpha_{B}=20^{\circ}$

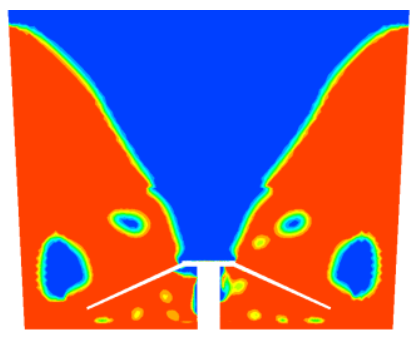

(d) $\alpha_{B}=26^{\circ}$

vater.Volume Fraction

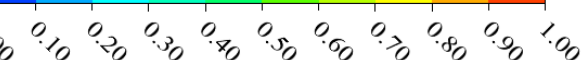

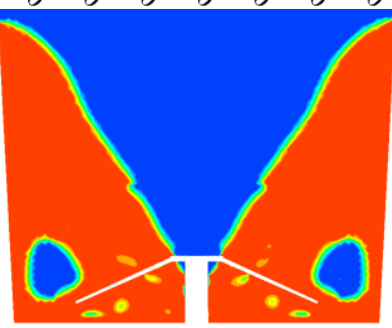

(b) $\alpha_{B}=22^{\circ}$

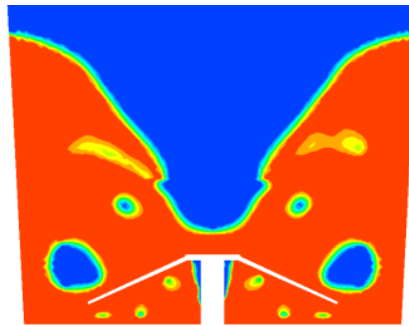

(e) $\alpha_{B}=28^{\circ}$

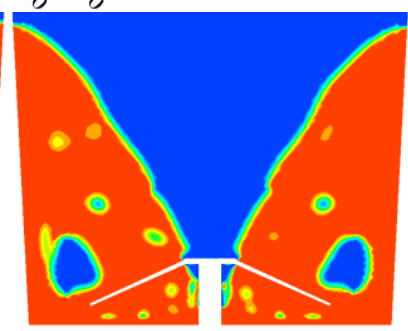

(c) $\alpha_{B}=24^{\circ}$

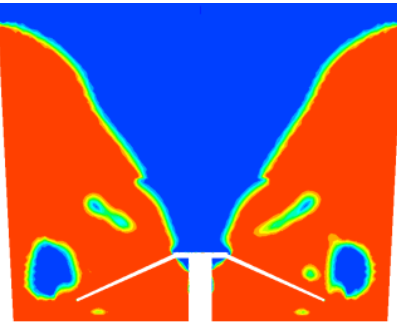

(f) $\alpha_{B}=30^{\circ}$

Fig. 14. Two-phase fluids distribution at the local X-Y plane at different $\alpha_{B}$.

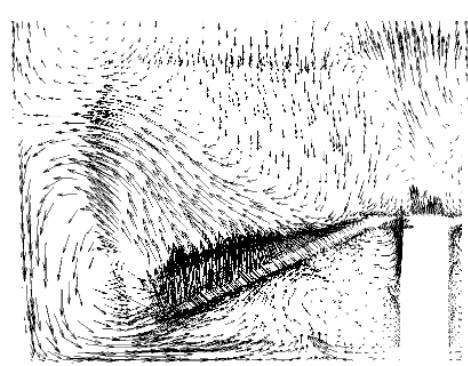

(a) $\alpha_{B}=20^{\circ}$

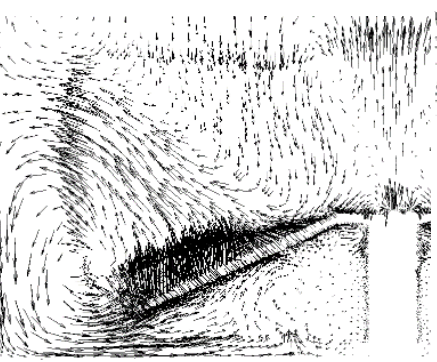

(b) $\alpha_{B}=22^{\circ}$

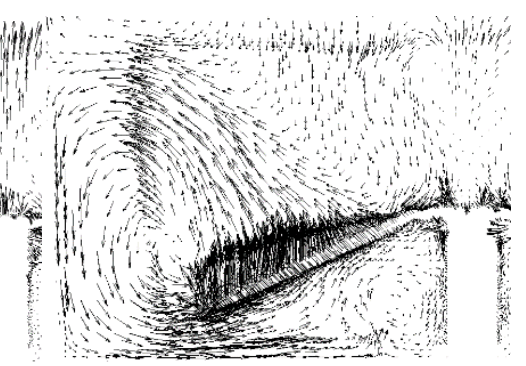

(c) $\alpha_{B}=24^{\circ}$

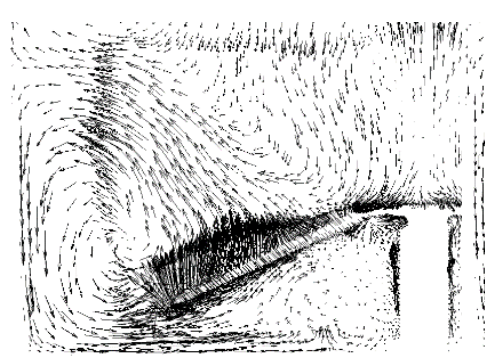

(d) $\alpha_{B}=26^{\circ}$

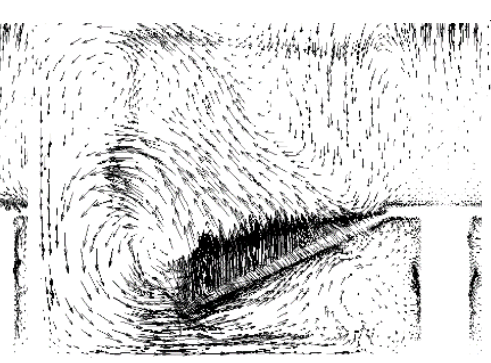

(e) $\alpha_{B}=28^{\circ}$

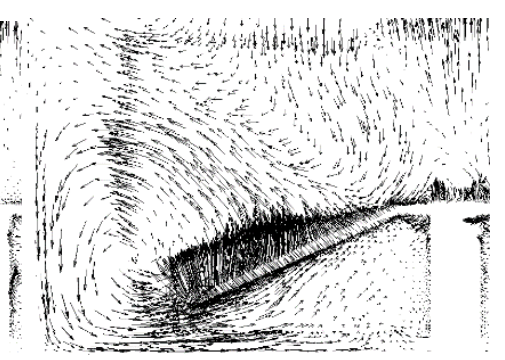

(f) $\alpha_{B}=30^{\circ}$

Fig. 15. Local velocity vector on $\mathrm{X}-\mathrm{Y}$ plane at different $\alpha_{B}$.

milk machine has been obtained. The main conclusions can be drawn as follow.

1. The inclination angle of the blade $\mathrm{A}, \alpha_{A}$, has a great influence on the gas-liquid fraction distribution, while the inclination angle of the blade $\mathrm{B}, \alpha_{B}$, has a small influence on the two-phase fraction distribution.

2. The inclination angle of the blade $\mathrm{A}, \alpha_{A}$, has a great influence on the velocity vector distribution of the flow field at the bottom of the cavity, while $\alpha_{B}$ has almost no effect on that. As $\left|\alpha_{A}\right|$ increases, the peak value of radial velocity decreases and moves to the interior of the cavity, so does the total pressure, which reduces the convective capacity of the bottom flow field. When $\alpha_{A}$ changes from $-24^{\circ}$ to $-26^{\circ}$, the velocity vector at the bottom of the 


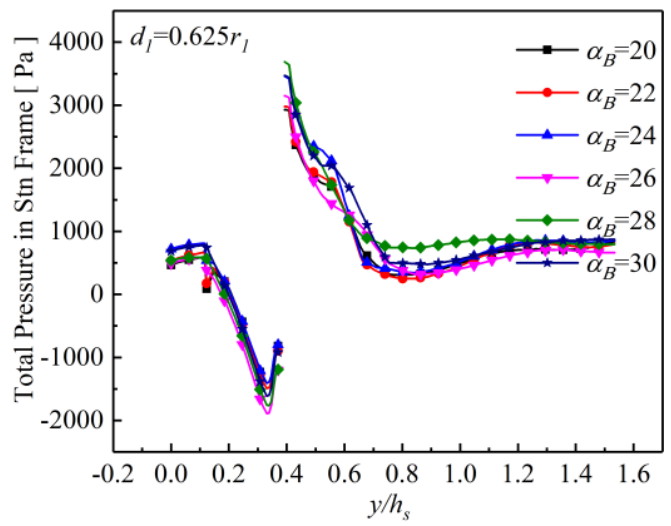

(a)

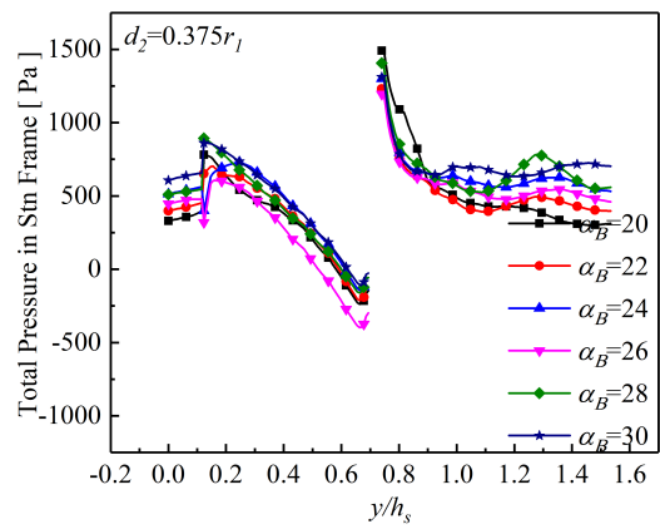

(b)

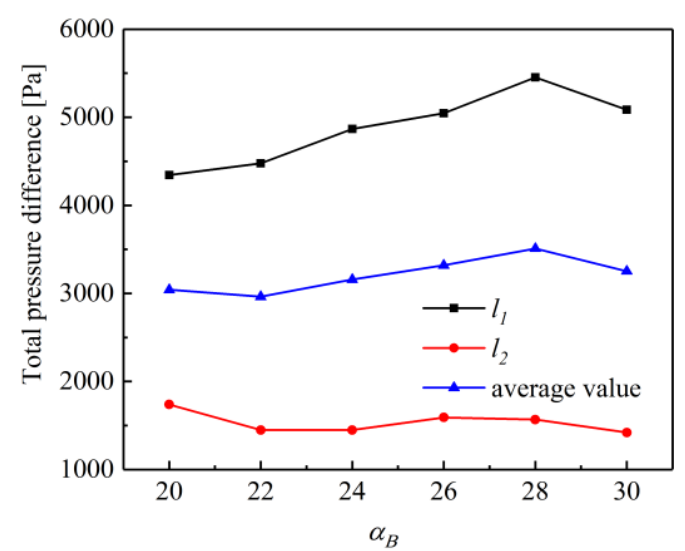

(c)

Fig. 16. Quantitative analysis of total pressure across A blade at different $\alpha_{B}$. (a) Total pressure variation on $l_{1}$; (b) Total pressure variation on $l_{2}$; (c) Variation of the maximum pressure difference of $l_{1}$ and $l_{2}$ and its average value.

cavity changes from the connected state to the separated state, this change of flow topology weakens the ability of the particles crushing. Thus, the best inclination angle of the blade $\mathrm{A}, \alpha_{A}$, should be between $-24^{\circ}$ and $-26^{\circ}$.

3. With the increase of $\left|\alpha_{A}\right|$, the average pressure difference between the up and the bottom surface of the blade A first increases and then decreases. At $\alpha_{A}=-24^{\circ} \sim-26^{\circ}$, the pressure difference increases sharply. The angle of blade $\mathrm{B}, \alpha_{B}$, has a certain effect on the pressure difference between the up and the bottom surface of blade A. Generally, as $\alpha_{B}$ increases, the pressure difference first increases and then decreases. At $\alpha_{B}=28^{\circ}$, the pressure difference across blade $\mathrm{A}$ is the largest, which is the most suitable value for efficient crushing. .

4. The inclination angle of the blade $\mathrm{A}, \alpha_{A}$, has a great influence on the radial and circumferential flow characteristics. Generally speaking, around $\alpha_{A}=-24^{\circ}$, the turbulent kinetic energy and eddy dissipation reach the maximum.
In summary, the best inclination anglesare $\alpha_{\text {Aopt }}=-24^{\circ} \sim-26^{\circ}\left(\alpha_{\text {Aopt }}<26^{\circ}\right)$ and $\alpha_{\text {Bopt }}=28^{\circ}$, respectively.

\section{ACKNOWLEDGMENT}

The authorswant to thank the help in the discussion from H.Guo and H. Huang in Joyoung Group Company. This research is supported by the research fund by Zhejiang Sc-Tech University (21022094-Y).

\section{REFERENCES}

Buffo, M. M., L. J. Corrêa, M. N. Esperanca, A. J. G. Cruz, C. S. Farinas and A. C. Badino (2016). Influence of dual-impeller type and configuration on oxygen transfer, power consumption, and shear rate in a stirred tank bioreactor. Biochemical Engineering Journal 114, 130-139.

Gradov, D. V., A. Laari, I. Turunen and T. Koiranen (2017). Experimentally validated CFD model 
for gas-liquid flow in a round-bottom stirred tank equipped with Rushton turbine. International Journal of Chemical Reactor Engineering 15(2).

Guini, Y. (2014). Numerical simulation of multi-layer agitator blade in high temperature floating two-phase flow field. Biotechnology: An Indian Journal 10(24):15984-15992.

Jia, H. L., F. Wang, J. X. Wu, X. Tan and M. Li (2020). CFD research on the influence of $45^{\circ}$ disk turbine agitator blade diameter on the solid-liquid mixing characteristics of the cone-bottom stirred tank. Arabian Journal for Science and Engineering 45, 5741-5749.

Kerdouss, F., A. Bannari and P. Proulx (2006). CFD modeling of gas dispersion and bubble size in a double turbine stirred tank. Chemical Engineering Science 61(10), 3313-3322.

Khopkar, A. R., A. R. Rammohan, V. V. Ranade and M. P. Dudukovic (2005). Gas-liquid flow generated by a Rushton turbine in stirred vessel: CARPT/CT measurements and CFD simulations. Chemical Engineering Science 60(8-9), 2215-2229.

Lane, G. L., M. P. Schwarz and G. M. Evans (2002). Predicting gas-liquid flow in a mechanically stirred tank. Applied Mathematical Modelling 26(2), 223-235.

Li, L., H. S. Dou, Y. Du, H. W. Guo and H. Huang (2020). Study on critical speed of vortex rupture and gas-liquid two-phase flow in rotating soybean milk machine. International Journal of Food Engineering 17(2), 97-110.

Li, L.C., J. J. Wang, X. P. Gu, L. F. Feng, and B. G. $\mathrm{Li}$ (2010). Computational fluid dynamics simulation of bubble size andlocal gas holdup in stirred vessel. Journal of Zhejiang University (Engineering Science) 044 (012), 2396-2400, 2415.

Li, X. J., X. P. Guan, N. Yang and M. Y. Liu (2017). Experimental study and CFD simulation of gas-liquid flow in a stirred tank using the EMMS drag model. Chemical Industryand Engineering Progress 36(11), 4000-4009.

Prakash, B., T. Bhatelia, D, Wadnerkar, M. T. Shah, V. K. Pareek and R. P. Utikar (2019). Vortex shape and gas-liquid hydrodynamics in unbaffled stirred tank. The Canadian Journal of Chemical Engineering 97(6), 1913-1920.

Ren, P. F. and J. Nan (2015). CFD and experimental studies on the impact of impeller type on flow field and floc size evolution in a stirred tank. International Conference on Advances in Energy \& Environmental Science 1473-1481.

Sanaie-Moghadam, M., M. Jahangiri and F. Hormozi (2015). Determination of stationary region boundary in multiple reference frames method in a mixing system agitated by helical ribbon impeller using CFD. Journal of Heat and Mass Transfer Research 2(01), 31-37.

Shao, X. M., X. L. Liu and Y. L. Li (2010). Experimental study of influence of the impeller spacing on flow behaviour of double-impeller stirred tank. The Canadian Journal of Chemical Engineering 81(6), 1239-1245.

Song, Q., X. B. Ze and X. Han (2017). Study of the flow field in household soymilk maker in relation to spoiler ribs quantity and location. International Conference on Mechanics and Mechatronics 5, 98-101.

Thakur, S, and J. Wright (2003) CFD Predictions of Turbomachinery Flows Using Quasi-Steady and Unsteady Models. Aiaa Fluid Dynamics Conference \& Exhibit.

Tian, L., Y. Liu, J. J. Tang, G. Z. Lv and T. A. Zhang (2017). Variation law of gas holdup in an autoclave during the pressure leaching process by using a mixed-flow agitator. International Journal of Minerals Metallurgy and Materials 24(8), 876-883.

Wang, G., L .J. Zhou, S. B. Xiong, Q. R. Li, M. C. $\mathrm{Wu}$ and S. M. Zhao (2011). Effects of blender structure and its force of soybean milk machine on soybean milk quality. Food Science 32(7), 162-167.

Wang, J. J., L. C. Li, X. P. Gu and L. F. Feng (2012). Progress on CFD simulation of gas-liquid two-phase flow in stirred tank reactor. Process Equipment \& Piping 49 (001), 1-4.

Wu, M. C., C. S. Wu, J. Y. Liang, Y. T. Lei and B. Yang (2017). Study on shear properties of six kinds of conventional agitator. Chemical Engineering (China) 45(08), 68-73.

Wu, Y., B. C. Ong and M. H. Al-Dahhan (2001). Predictions of radial gas holdup profiles in bubble column reactors. Chemical Engineering Science 56(3), 1207-1210.

Zhang, Y. Q., X. Pan, Y. H. Wang, P. C. Luo and H. $\mathrm{Wu}$ (2018). Numerical and experimental investigation on surface air entrainment mechanisms of a novel long-short blades agitator. AIChE Journal 64(1), 316-325.

Zhang, Y., Y. Yong, Z. S. Mao, C. Yang, H. Sun, H. Wang and C. Yang (2010). Numerical simulation of gas-liquid flow in a stirred tank with swirl modification. Chemical Engineering \& Technology 32(8), 1266-1273.

Zhou, C., H. W. Zhao and J. K. Zheng (2013). Impact of Blade Installing Angle on Open Turbine Stirrer. Machine Building \& Automation 41(1), 27-30. 